\title{
Isolation of proximity-induced triplet pairing channel in a superconduc- tor/ferromagnet spin valve
}

\author{
Andrey Kamashev ${ }^{1, \star}$, Aidar Validov ${ }^{1}$, Nadir Garif'yanov ${ }^{1}$, Yakov Fominov 2,3 , Pavel Leksin ${ }^{4}$, Joachim Schumann ${ }^{4}$, \\ Jürgen Thomas ${ }^{4}$, Vladislav Kataev ${ }^{4}$, Bernd Büchner ${ }^{4,5}$, and Ilgiz Garifullin ${ }^{1}$ \\ ${ }^{1}$ Zavoisky Physical-Technical Institute, Russian Academy of Sciences, 420029 Kazan, Russia \\ ${ }^{2}$ L.D. Landau Institute for Theoretical Physics, Russian Academy of Sciences, Chernogolovka, Russia \\ ${ }^{3}$ Moscow Institute of Physics and Technology, 141700 Dolgoprudny, Russia \\ ${ }^{4}$ Leibniz Institute for Solid State and Materials Research IFW Dresden, D-01069 Dresden, Germany \\ ${ }^{5}$ Institute for Solid State Physics, Technical University Dresden, D-01062 Dresden, Germany
}

\begin{abstract}
In the present work we have studied the proximity-induced superconducting triplet pairing in $\mathrm{CoO}_{x} / \mathrm{Py} 1 / \mathrm{Cu} / \mathrm{Py} 2 / \mathrm{Cu} / \mathrm{Pb}$ spin-valve structure (where $\mathrm{Py}=\mathrm{Ni}_{0.81} \mathrm{Fe}_{0.19}$ ). For $\mathrm{CoO}_{x}(3 \mathrm{~nm}) / \mathrm{Py}(3 \mathrm{~nm}) / \mathrm{Cu}(4 \mathrm{~nm}) / \mathrm{Py}(0.6 \mathrm{~nm}) / \mathrm{Cu}(2 \mathrm{~nm}) / \mathrm{Pb}(70 \mathrm{~nm})$ we have studied the dependence of the $T_{c}$ on the angle $\alpha$ between the direction of the cooling field and the external field both applied in the plane of the sample. We obtained that the $T_{c}$ does not change monotonically with the angle but passes through a minimum. To observe an "isolated" triplet spin-valve effect we exploited the oscillatory feature of the magnitude of the ordinary spin-valve effect $\Delta T_{c}$ in the dependence of the Py2-layer thickness $d_{P y 2}$. We determined the value of $d_{P y 2}$ at which $\Delta T_{c}$ caused by the ordinary spin-valve effect is suppressed. This means that the difference in the $T_{c}$ between the antiparallel and parallel mutual orientation of magnetizations of the Py1 and Py2 layers is zero. For such a sample a "pure" triplet spin-valve effect which causes the minimum in $T_{c}$ at the orthogonal configuration of magnetizations has been observed.
\end{abstract}

\section{Introduction}

The superconducting spin-valve effect consists in the fact that the degrees of suppression of superconductivity in thin-film multilayer structures $\mathrm{F} 1 / \mathrm{F} 2 / \mathrm{S}$ or F1/S/F2 are different for the parallel $(\mathrm{P})$ and antiparallel $(\mathrm{AP})$ relative orientations of the magnetizations of the F1 and F2 ferromagnetic layers [1]. The operation of the superconducting spin valve based on the superconductor/ferromagnet $(\mathrm{S} / \mathrm{F})$ proximity effect provides a key to the understanding of many fundamental aspects of the relationship between superconductivity and magnetism. Spin valves have good prospects for use as passive elements of superconducting spintronic devices and should operate with a change of the direction of a weak external magnetic field. The manifestation of this effect in S/F systems with a good contact between the metallic ferromagnetic and superconducting layers prepared from ordinary metals and conventional ferromagnets was confirmed in a large number of experimental studies (see, for example, recent reviews [2-4] and references therein). Recent theoretical studies (see, for example,reviews [2-8]) have predicted the generation of a long-range triplet component of a superconducting condensate in S/F-structures. As follows from the theory proposed by Fominov et al. [9], the presence of a minimum of the superconducting transition temperature $T_{c}$ in

\footnotetext{
^e-mail:kamandi@mail.ru
}

the vicinity of the orthogonal configuration of the magnetizations clearly indicates the generation of the longrange triplet component. We have experimentally confirmed the existence of the long-range triplet component for the $\mathrm{CoO}_{x} / \mathrm{Fe} 1 / \mathrm{Cu} / \mathrm{Fe} 2 / \mathrm{Pb}$ superconducting spin-valve structure [10]. The observed angular dependence of the superconducting transition temperature $T_{c}$ is caused by a combination of the singlet and triplet components of the superconducting condensate. The existence of the triplet contribution to the superconducting spin-valve effect has also been shown in [11-15]. An important question for basic and applied research in the field of spintronics is whether there is the possibility of observing the "isolated" triplet contribution to the superconducting spin-valve effect. At first glance, this seems to be impossible, because the long-range triplet components arise from the singlet components and cannot exist without them. However, in this study, we have experimentally observed the "isolated" triplet effect caused by the oscillatory behavior of the dependence $\Delta T_{c}\left(d_{F 2}\right)$, which effectively suppresses the conventional superconducting spin-valve effect with an appropriate choice of the thickness of the $\mathrm{F} 2 d_{F 2}$ layer.

\section{Samples}

To investigate the proximity-induced triplet pairing we measured the magnitude of the spin-valve effect in 
the dependence of the Py2-layer thickness for the $\mathrm{CoO}_{x} / \mathrm{Py} 1 / \mathrm{Cu} / \mathrm{Py} 2 / \mathrm{Cu} / \mathrm{Pb}$ multilayer grown on the $\mathrm{MgO}$ (001) substrate. Here Py denotes permalloy $\mathrm{Ni}_{0.81} \mathrm{Fe}_{0.19}$. Optimization of the preparation conditions of the samples and their characterization are described in [16]. The optimal thickness of the $\mathrm{Pb}$ layer $d_{P b}=70 \mathrm{~nm}$ was determined from the $T_{c}\left(d_{P b}\right)$ curve measured at a constant $d_{P y 1}=5 \mathrm{~nm}$, which is much larger than the penetration depth $\xi_{h}$ of Cooper pairs into ferromagnetic Py. Basing on our data on $T_{c}\left(d_{P y}\right)$ at fixed $d_{P b}$ we estimate this value as $\xi_{h} \sim 1.1 \mathrm{~nm}$.

Earlier we revealed that the F1-layer thickness at a fixed $d_{F 2}$ does not significantly influence $\Delta T_{c}$ for the sample set $\mathrm{CoO}_{x}(2.5 \mathrm{~nm}) / \mathrm{Fe} 1\left(d_{F e 1}\right) / \mathrm{Cu}(4 \quad \mathrm{~nm}) / \mathrm{Fe} 2\left(d_{F e 2}\right) / \mathrm{Cu}(1.2$ $\mathrm{nm}) / \mathrm{Pb}(60 \mathrm{~nm})$ and that a thin $\mathrm{Cu}(1.2 \mathrm{~nm})$ interlayer between F2- and S- layers is completely transparent for the Cooper pairs [17].

\section{Experimental results}

We investigated the transport properties of samples. We analyzed the dependence of the superconducting transition temperature $T_{c}$ on the angle $\alpha$ between the magnetizations of the Py1 and Py2 ferromagnetic layers in the magnetic field $H_{0}=+100$ Oe applied in the plane of the sample. The most interesting results on the dependence $T_{c}(\alpha)$ were obtained for the structures $\mathrm{CoO}_{x} / \mathrm{Py}(3 \mathrm{~nm}) / \mathrm{Cu}(4 \mathrm{~nm}) / \mathrm{Py}(0.6 \mathrm{~nm}) / \mathrm{Cu}(2 \mathrm{~nm}) / \mathrm{Pb}(70 \mathrm{~nm})$ (sample 1) and $\mathrm{CoO}_{x} / \mathrm{Py}(3 \mathrm{~nm}) / \mathrm{Cu}(4 \mathrm{~nm}) / \mathrm{Py}(3 \mathrm{~nm}) / \mathrm{Cu}(2$ $\mathrm{nm}) / \mathrm{Pb}(70 \mathrm{~nm}$ ) (sample 2) (see Fig 1). As can be seen from Fig. 1, the angular dependences of the superconducting transition temperatures $T_{c}$ of these samples have a nonmonotonic behavior. The dependence $T_{c}(\alpha)$ passes through a minimum in the range between the parallel $(\alpha=$ $\left.0^{\circ}\right)$ and antiparallel $\left(\alpha=180^{\circ}\right)$ orientations in the vicinity of the orthogonal configuration of the magnetizations. For sample 1, the magnitude of the superconducting spinvalve effect $\Delta T_{c}$ exceeds the width of the superconducting transition with a change in the relative orientation of the magnetizations from the antiparallel to orthogonal orientation. Consequently, in this structure, there is the possibility of the complete turn-on/turn-off switching of the superconducting current. In this case, we observed the complete turn-on/turn-off switching of the superconducting current due to a combination of the conventional and triplet spin-valve effects.

In the samples under investigation, the long-range triplet component of the superconducting condensate contributes to the superconducting spin-valve effect. However, the triplet contribution to the spin-valve effect coexists with the contribution from the conventional component of the condensate. As was shown previously, the amplitude of the conventional superconducting spin-valve effect can be suppressed to zero for a particular thickness of the second ferromagnet layer due to the oscillatory behavior of the dependence $T_{c}\left(d_{F 2}\right)[18,19]$. These oscillations are caused by the interference of the pair wave function at the F2/S interface with the wave function incident on it and reflected from the F1/F2 interface.

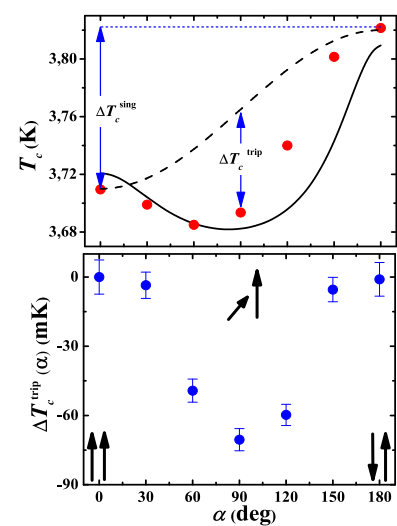

(a)

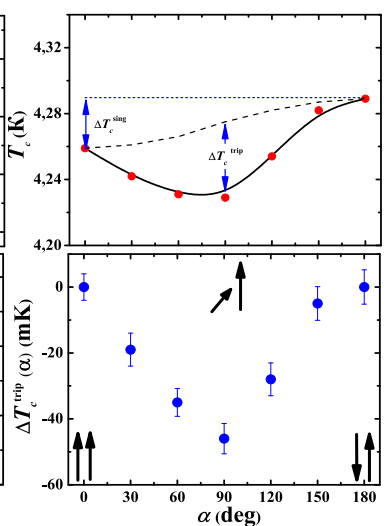

(b)
Figure 1. Dependences of the superconducting transition temperatures on the angle between the magnetizations of the ferromagnetic layers in samples (a) 1 and (b) 2. The upper panels show the angular dependences of the temperature $T_{c}$ measured in the external magnetic field $H_{0}=+100 \mathrm{Oe}$ (circles). The dashed lines are the reference curves (see formula (1)). The solid lines are the theoretical curves calculated according to the theory proposed by Fominov and his colleagues (see [10]). The lower panels show the angular dependences of the quantity $\Delta T_{c}{ }^{t r i p}$, i.e., the difference between the actual value of the temperature $T_{c}$ and the reference curve for the corresponding samples.

For the $\mathrm{CoO}_{x} / \mathrm{Fe} 1 / \mathrm{Cu} / \mathrm{Fe} 2 / \mathrm{Cu} / \mathrm{Pb}$ structure, the superconducting spin-valve effect $\Delta T_{c}$ vanishes in the range of thicknesses $d_{F e 2} \approx 0.8-1.0 \mathrm{~nm}[17,20]$. From the analysis of the data on the dependence $T_{c}\left(d_{P y}\right)$, it was concluded that the depth of penetration of the Cooper pairs into the permalloy layer is two times greater than the value obtained for iron. This means that the equality $\Delta T_{c}=0$ for the samples with permalloy should be achieved for layer thicknesses in the range $d_{P y 2} \approx 1.5-1.7 \mathrm{~nm}$. Indeed, for the $\mathrm{CoO}_{x} / \mathrm{Py}(3 \mathrm{~nm}) / \mathrm{Cu}(4 \mathrm{~nm}) / \mathrm{Py}(1.7 \mathrm{~nm}) / \mathrm{Cu}(2 \mathrm{~nm}) / \mathrm{Pb}(70 \mathrm{~nm})$ structure (sample 3), we observed an isolated triplet contribution to the superconducting spin-valve effect (see Fig. 2). The angular dependence $T_{c}(\alpha)$ for this sample has the form of a curve in which the critical temperatures $T_{c}$ at $\alpha=0^{\circ}$ and $\alpha=180^{\circ}$ coincide with each other (the conventional superconducting spin-valve effect $\left.\Delta T_{c}=\Delta T_{c}{ }^{A P}-\Delta T_{c}{ }^{A P}=0\right)$. At the same time, when the configuration of the magnetizations of the Py1 and Py2 layers is noncollinear, the dependence $T_{c}(\alpha)$ has a minimum. A comparison of these data with the theory for the same parameters as for the description of the data obtained for the samples in Fig. 1 demonstrated a satisfactory agreement (see the solid line in Fig. 2). The only difference was in the values of $d_{P y 2}$, which was taken to be equal to $1.7 \mathrm{~nm}$. Thus, it was demonstrated that there is the possibility of the isolation (separate observation) of the triplet contribution to the superconducting spin-valve effect. The creation of an S/F heterostructure, where the contribution from the singlet component to the superconducting spin-valve effect is ineffective in terms of the influence on the critical temperature $T_{c}$ due to 
the interference of pair wave functions, is an interesting problem.

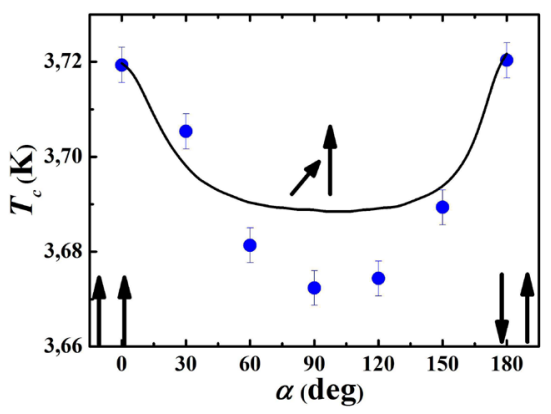

Figure 2. Sample of the $\mathrm{CoO}_{x} / \mathrm{Py}(3 \mathrm{~nm}) / \mathrm{Cu}(4 \mathrm{~nm}) / \mathrm{Py}(3 \mathrm{~nm}) / \mathrm{Cu}(2 \mathrm{~nm}) / \mathrm{Pb}(70 \mathrm{~nm})$ structure (sample 3) with zero conventional spin-valve effect $\left(\Delta T_{c}{ }^{P}=\Delta T_{c}{ }^{A P}\right)$. The circles show the angular dependence of the superconducting transition temperature $T_{c}(\alpha)$, which is caused by the long-range triplet component of the superconducting condensate. The solid line is the theoretical curve.

\section{Discussion of experimental results}

As can be seen from Fig. 1, the magnitude of the conventional superconducting spin-valve effect is equal to $\Delta T_{c}$ $=110 \mathrm{mK}$ for sample 1 and $\Delta T_{c}=30 \mathrm{mK}$ for sample 2 . This difference in magnitude of the spin-valve effect $\Delta T_{c}$ is associated with the fact that, for the thickness of the permalloy layer $d_{P y 2}=3 \mathrm{~nm}$ in sample 2 , the wave function of superconductor Cooper pairs almost completely decays in the Py2 ferromagnetic layer. Therefore, the layer Py1 has little influence on the superconductivity and, consequently, on the magnitude of the superconducting spinvalve effect $\Delta T_{c}$. This is consistent with the fact that, in accordance with the theory, a decrease in the thickness of the permalloy layer $d_{P y 2}$ leads to an increase in the superconducting spin-valve effect $\Delta T_{c}$. It can also be seen from Fig. 1 that, when the relative orientation of the magnetizations changes from the parallel (P) $\left(\alpha=0^{\circ}\right)$ to antiparallel (AP) $\left(\alpha=180^{\circ}\right)$ orientation, the superconducting transition temperature $T_{c}$ varies monotonically, passing through the minimum for the noncollinear orientation. According to the theory (see [10]), the characteristic minimum in the dependence $T_{c}(\alpha)$, which is most pronounced in the vicinity of the angle $\alpha=90^{\circ}$, clearly indicates the generation of the long-range triplet component in the superconducting condensate. By assuming that there is no triplet component (even though, according to the theory, the appearance of such a component is inevitable), it can be expected that the dependence $T_{c}(\alpha)$ will be monotonic. From the general considerations, the superconducting transition temperature $T_{c}$ should be a function of the quantities $\alpha^{2}$ and $(\pi-\alpha)^{2}$ for the angle $\alpha$ varying from 0 to $\pi$. Thus, the dependence $T_{c}(\alpha)$ can be expressed in terms of the temperatures and as follows:

$$
T_{c}^{r e f}(\alpha)=T_{c}^{P} \cos ^{2}(\alpha / 2)+T_{c}^{A P} \sin ^{2}(\alpha / 2) .
$$

This imaginary curve is represented by the dashed line in Fig. 1 for the dependences $T_{c}(\alpha)$. This curve will be referred to as the reference curve. The deviation of the actual temperature $T_{c}$ from the reference curve is shown in Fig. 1 for the dependences $\Delta T_{c}{ }^{\text {trip }}(\alpha)$. The value of this deviation demonstrates the contribution from the long-range triplet component to the magnitude of the superconducting spin-valve effect. Let us designate this magnitude as $\Delta T_{c}{ }^{\text {trip }}$ and find that the difference in the temperatures $T_{c}$ between the antiparallel and perpendicular orientations of the magnetizations already is equal to $130 \mathrm{mK}$ for sample 1 and $60 \mathrm{mK}$ for sample 2 . This means that the long-range triplet component makes a significant contribution to the superconducting spin-valve effect.

In all previous studies of our group (see [10, 17-20]), the experimental data on the critical temperature $T_{c}$ were compared with the parameter of the theory $W$, which determines the degree of suppression of superconductivity by the ferromagnetic layer (see [7]). The effective boundary condition for the real component of the anomalous Green's function can be written as

$$
\xi_{S} \frac{d f_{0}}{d x}=W f_{0} .
$$

This analysis demonstrated that there is a qualitative agreement between the theory (parameter $W$ ) and the experiment $\left(T_{c}\right)$ without calculating the critical temperature itself. Here, for the first time, we carried out a direct comparative analysis of the theoretically calculated and experimentally measured values of the superconducting transition temperature $T_{c}$. For the theoretical calculation of the dependence $T_{c}(\alpha)$, we used the theory proposed by Fominov and his colleagues (see [9]) for the case of an arbitrary transparency of the F2/S interface (see $[17,20]$ ). The result of this calculation is represented by the solid line in Fig. 1. The theoretical calculation was performed with the following parameters: the coherence lengths for the S- and F-layers were taken as $\xi_{S}=41 \mathrm{~nm}$ and $\xi_{F}=13 \mathrm{~nm}$, respectively; the thickness of the superconducting layer was $d_{P b}=73.5 \mathrm{~nm}$; the temperature of the transition to the superconducting state of bulk lead was $\Delta T_{c}{ }^{P b}=7.2 \mathrm{~K}$; the transparency parameters at the $\mathrm{Py} 2 / \mathrm{Pb}$ interface were $\gamma=$ 0.734 and $\gamma_{b}=1.8$; and the exchange field in the ferromagnet was $h=0.3 \mathrm{eV}$. In the theoretical calculations, it was found that, according to the theory, the thickness of the Py2 layer $d_{P y 2}$ should be two times less than the actual value of the layer thickness. This is likely associated with the fact that the Py2 layer is surrounded on both sides by copper layers, and the effective thickness of the Py2 layer decreases due to the mutual diffusion of the copper atoms and permalloy. As can be seen from Figs. 1 and 2 , the theory reproduces the main features of the experimental dependences. The significant discrepancy between theory and experiment can be caused by two factors. In the theory proposed by Fominov et al. [9], the F-layers were represented by weak ferromagnets, whereas permalloy is not ferromagnetic. In addition, we ignored the limitation of the transparency of the $\mathrm{Py} 1 / \mathrm{Cu} / \mathrm{Py} 2$ interface, as was done in the theoretical studies of Deminov et al. [21, 22], because, in our case, this parameter would be free. In 
contrast to the parameters characterizing the transparency of the $\mathrm{Py} 2 / \mathrm{Pb}$ interface, we cannot estimate this quantity from the experiment.

\section{Conclusions}

Thus, the main results obtained in the investigations presented in this paper can be summarized as follows. First, the long-range triplet component in $\mathrm{CoO}_{x} / \mathrm{Py} 1 / \mathrm{Cu} / \mathrm{Py} 2 / \mathrm{Cu} / \mathrm{Pb}$ superconducting spin valve samples has been studied experimentally. It has been revealed that there is a complete switching between the normal and superconducting states. The switching is caused by a combination of the conventional and triplet spin-valve effects when the relative orientation of the magnetizations of the Py1 and Py2 layers changes from the antiparallel to perpendicular orientation. Second, a direct comparison of the theoretically calculated and experimentally obtained values of the superconducting transition temperature $T_{c}$ has been performed for the first time.

This work was partially supported by Russian Foundation for Basic Research (No. 17-02-00229) and the Program of the Russian Academy of Sciences.

\section{References}

[1] S. Oh, D. Youm, M.R. Beasley, Appl. Phys. Lett. 71, 2376 (1997)

[2] M. Eschrig, Physics Today 64, 43 (2011)

[3] M.G. Blamire, J.W.A. Robinson, J. Phys: Condens. Matter 26, 453201 (2014)

[4] J. Linder, J.W.A. Robinson, Nat. Phys. 11, 307 (2015)

[5] F.S. Bergeret, A.F. Volkov, K.B. Efetov, Rev. Mod. Phys. 77, 1321 (2005)

[6] A.I. Buzdin, Rev. Mod. Phys. 77, 935 (2005)

[7] K.B. Efetov, I.A. Garifullin, A.F. Volkov, K. Westerholt, Magnetic Heterostructures. Advances and Perspectives in Spinstructures and Spintransport, Vol. 227 of Springer Tracts in Modern Physics (Springer Berlin Heidelberg, 2007)

[8] K.B. Efetov, I.A. Garifullin, A.F. Volkov, K. Westerholt, Magnetic Nanostructures. Spin Dynamic and SpinTransport., Vol. 246 of Springer Tracts in Modern Physics (Springer Berlin Heidelberg, 2013)
[9] Y.V. Fominov, A.A. Golubov, T.Y. Karminskaya, M.Y. Kupriyanov, R.G. Deminov, L.R. Tagirov, JETP Letters 91, 308 (2010)

[10] P.V. Leksin, N.N. Garif'yanov, I.A. Garifullin, Y.V. Fominov, J. Schumann, Y. Krupskaya, V. Kataev, O.G. Schmidt, B. Büchner, Phys. Rev. Lett. 109, 057005 (2012)

[11] V.I. Zdravkov, J. Kehrle, G. Obermeier, D. Lenk, H.A. Krug von Nidda, C. Müller, M.Y. Kupriyanov, A.S. Sidorenko, S. Horn, R. Tidecks et al., Phys. Rev. B 87, 144504 (2013)

[12] A.A. Jara, C. Safranski, I.N. Krivorotov, C.T. Wu, A.N. Malmi-Kakkada, O.T. Valls, K. Halterman, Phys. Rev. B 89, 184502 (2014)

[13] X.L. Wang, A. Di Bernardo, N. Banerjee, A. Wells, F.S. Bergeret, M.G. Blamire, J.W.A. Robinson, Phys. Rev. B 89, 140508(R) (2014)

[14] N. Banerjee, C.B. Smiet, R.G.J. Smits, A. Ozaeta, F.S. Bergeret, M.G. Blamire, J.W.A. Robinson, Nat.Commun. 5, 3048 (2014)

[15] M.G. Flokstra, T.C. Cunningham, J. Kim, N. Satchell, G. Burnell, P.J. Curran, S.J. Bending, C.J. Kinane, J.F.K. Cooper, S. Langridge et al., Phys. Rev. B 91, 060501 (2015)

[16] P.V. Leksin, A.A. Kamashev, J. Schumann, V. Kataev, J. Thomas, B. Büchner, I.A. Garifullin, Nano Research 9, 1005 (2016)

[17] P.V. Leksin, N.N. Garif'yanov, A.A. Kamashev, Y.V. Fominov, J. Schumann, C. Hess, V. Kataev, B. Büchner, I.A. Garifullin, Physical Review B 91, 214508 (2015)

[18] P. Leksin, N. Garif'yanov, I. Garifullin, J. Schumann, V. Kataev, O. Schmidt, B. Büchner, Phys. Rev. Lett. 106, 067005 (2011)

[19] P.V. Leksin, N.N. Garif'yanov, I.A. Garifullin, J. Schumann, V. Kataev, O.G. Schmidt, B. Büchner, Phys. Rev. B 85, 024502 (2012)

[20] P.V. Leksin, A.A. Kamashev, N.N. Garif'yanov, I.A. Garifullin, Y.V. Fominov, J. Schumann, C. Hess, V. Kataev, B. Büchner, JETP 97, 478 (2013)

[21] R. Deminov, L. Tagirov, R. Gaifullin, T. Karminskay, M. Kupriyanov, Y. Fominov, A. Golubov, Journal of Magnetism and Magnetic Materials 373, 16 (2015)

[22] R. Deminov, L. Tagirov, R. Gaifullin, T. Karminskay, M. Kupriyanov, Y. Fominov, A. Golubov, Solid State Phenomena 233-234, 745 (2015) 